\title{
On a Singular Problem of Cauchy-Nicoletti (*).
}

\author{
I. T. KIGURADZE (Tbilisi, U.S.S.R.)
}

Summary. - The sufficient conditions of the existence and uniqueness of the solution of the problem

$$
\frac{d x_{i}}{d t}=f_{i}\left(t, x_{1}, \ldots, x_{n}\right), \quad x_{i}\left(t_{i}\right)=0 \quad(i=1, \ldots, n)
$$

are given for the case when the functions $f_{i}\left(t, x_{1}, \ldots, x_{n}\right)(i=1, \ldots, n)$ are in generally, nonsummable with respect to $t$.

Consider the system of ordinary differential equations

$$
\frac{d x_{i}}{d t}=f_{i}\left(t, x_{1}, \ldots, x_{n}\right) \quad(i=1, \ldots, n)
$$

with right sides defined on the domain

$$
\mathbb{D}_{a b}^{n}=\left\{\left(t, x_{1}, \ldots, x_{n}\right): a<t<b,-\infty<x_{1}, \ldots, x_{n}<+\infty\right\},
$$

where $-\infty<a<b<+\infty$.

For system (1) we are concerned with the following

Problem of Cauchy-Nicolett. - To find for given $t_{i} \in[a, b](i=1, \ldots, n)$, some functions absolutely continuous on the interval $[a, b]$ and satisfying almost everywhere on this interval the system (1) and the boundary conditions

$$
x_{i}\left(t_{i}\right)=0 \quad(i=1, \ldots, n)
$$

O. Nicoletri [9] was the first to investigate problem (1), (2). His results are generalized and specified in a number of works [6], [7], [11]-[13], [15], etc.

The above-mentioned works deal with the case when the right sides of system (1) are either continuous, or satisfy Caratheodory's conditions. In the present paper we study a singular case when the functions $f_{i}\left(t, x_{1}, \ldots, x_{n}\right)(i=1, \ldots, n)$ are, in general, nonsummable with respect to $t$.

Among earlier results in this direction mention should be made of the work by W. A. ČĚrK [13] in which a singular problem of Cauchy was studied for the first time, as well as the other papers, namely, [1]-[3], [5] and [10].

(*) Entrata in Redazione il 30 maggio 1973. 


\section{1. - Existence and uniqueness theorems for the system.}

I. - Throughout this paper we shall have the following definitions and notations:

1) $\bar{x}=\left(x_{i}\right)_{i=1}^{n}$ is the column-vector with the elements $x_{i}(i=1, \ldots, n)$, $A=\left(a_{i k}\right)_{i, k=1}^{n}$ means the $n \times n$ matrix with the elements $a_{i k}(i, k=1, \ldots, n)$,

$$
\begin{aligned}
& |\bar{x}|=\left(\left|x_{i}\right|\right)_{i=1}^{n}, \quad|A|=\left(\left|a_{i k}\right|\right)_{i, k=1}^{n}, \\
& \|\bar{x}\|=\sum_{i=1}^{n}\left|x_{i}\right|, \quad\|A\|=\sum_{i, k=1}^{n}\left|a_{i k}\right| .
\end{aligned}
$$

2) If $A=\left(a_{i k}\right)_{i, k=1}^{n}, B=\left(b_{i k}\right)_{i, k=1}^{n}, \bar{x}=\left(x_{i}\right)_{i=1}^{n}$ and $\bar{y}=\left(y_{i}\right)_{i=1}^{n}$, then the notation

$$
A \leqslant B \quad \text { and } \quad \bar{x} \leqslant \bar{y}
$$

means that

$$
a_{i k} \leqslant b_{i k}, \quad x_{i} \leqslant y_{i} \quad\left(i, z_{i}=1, \ldots, n\right)
$$

3) $L(a, b)$ is a space of functions which are summable on $(a, b)$.

4) Let $f\left(t, x_{1}, \ldots, x_{n}\right)$ be some function defined on the domain $\mathcal{D}_{a b}^{n} . f\left(t, x_{1}, \ldots, x_{n}\right) \in$ $\in K(a, b)$ means that the function $f\left(t, x_{1}, \ldots, x_{n}\right)$ satisfies Caratheodory's local conditions, i.e. this function is measurable in $t$ on $(a, b)$ for arbitrary $x_{1}, \ldots, x_{n}$, it is continuous in $x_{1}, \ldots, x_{n}$ on the domain $-\infty<x_{1}, \ldots, x_{n}<+\infty$ for almost all $t \in(a, b)$ and

$$
\sup \left\{\left|f\left(t, x_{1}, \ldots, x_{n}\right)\right|:\left|x_{i}\right| \leqslant \varrho(i=1, \ldots, n)\right\} \in L(a, b)
$$

for arbitrary $\varrho \in(0,+\infty)$.

5) By $K\left(a, b ; \tau_{1}, \ldots, \tau_{m}\right)$ with $\tau_{i} \in[a, b](i=1, \ldots, m)$ we shall denote the set of all functions belonging to $K(\alpha, \beta)$ for arbitrary $[\alpha, \beta] \subset[a, b]$ satisfying the condition

$$
\tau_{k} \notin[\alpha, \beta] \quad(k=1, \ldots, m) .
$$

In an analogous way we define $L\left(a, b ; \tau_{1}, \ldots, \tau_{m}\right)$ too.

6) The nonnegative matrix $A(t)=\left(a_{i k}(t)\right)_{i, k=1}^{n}$ is said to belong to the set $N_{0}\left(a, b ; t_{1}, \ldots, t_{n}\right)$, if $a_{i k}(t) \in L(a, b)(i, k=1, \ldots, m)$ and the system of differential inequalities

$$
\left|x_{i}^{\prime}(t)\right| \leqslant \sum_{k=1}^{n} a_{i k}(t)\left|x_{k}(t)\right| \quad \text { for } \quad a<t<b \quad(i=1, \ldots, n)
$$

has only the trivial solution satisfying boundary conditions (2). 
7) The vector-function $\bar{g}(t, \bar{x})=\left(g_{i}\left(t, x_{1}, \ldots, x_{n}\right)\right)_{i=1}^{n}$ is said to belong to the set $N\left(a, b ; t_{1}, \ldots, t_{n}\right)$ if every function $g_{i}\left(t, x_{1}, \ldots, x_{n}\right) \in \mathbb{K}(a, b)$ is nonnegative, nondecreasing in $x_{1}, \ldots, x_{i-1}, x_{i+1}, \ldots, x_{n}$ and for arbitrary $x_{0 k} \in(-\infty,+\infty)(k=1, \ldots, n)$ there exists a positive number $c_{0}=c_{0}\left(x_{01}, \ldots, x_{0 n}\right)$ such that

$$
\left|x_{i}(t)\right| \leqslant c_{0} \quad \text { for } a \leqslant t \leqslant b \quad(i=1, \ldots, n)
$$

for any funetions $x_{i}(t)(i=1, \ldots, n)$, absolutely continuous on $[a, b]$ and satisfying the conditions

$$
x_{i}\left(t_{i}\right)=x_{0 i}, \quad\left|x_{i}^{\prime}(t)\right| \leqslant g_{i}\left(t,\left|x_{1}(t)\right|, \ldots,\left|x_{n}(t)\right|\right) \quad \text { for } a<t<b(i=1, \ldots, n) .
$$

II. - Formulations of existence and uniqueness theorems.

THEOREM 1.1. - Let

$$
f_{i}\left(t, x_{1}, \ldots, x_{n}\right) \in K\left(a, b ; \tau_{i 1}, \ldots, \tau_{i m_{\ell}}\right) \quad(i=1, \ldots, n)
$$

and let on the domain $\mathfrak{D}_{a b}^{n}$ the inequalities

hold, where

$$
\begin{aligned}
& f_{i}\left(t, x_{1}, \ldots, x_{n}\right) \operatorname{sign}\left[\left(t-t_{i}\right)\left(x-x_{0 i}\right)\right] \leqslant \\
& \quad \leqslant-a_{i}(t)\left|x_{i}-x_{0 i}\right|+g_{i}\left(t,\left|x_{1}-x_{01}\right|, \ldots,\left|x_{n}-x_{0 n}\right|\right) \quad(i=1, \ldots, n)
\end{aligned}
$$

$$
\begin{gathered}
\left(g_{i}\left(t, x_{1}, \ldots, x_{n}\right)\right)_{i=1}^{n} \in N\left(a, b ; t_{1}, \ldots, t_{n}\right), \quad a_{i}(t) \geqslant 0, \\
a_{i}(t) \in L\left(a, b ; \tau_{i 1}, \ldots, \tau_{i m_{i}}\right), \quad a \leqslant \tau_{i k} \leqslant b \quad\left(k=1, \ldots, m_{i} ; i=1, \ldots, n\right), \\
x_{0 i}=0 \quad \text { for } t_{i} \in\left\{\tau_{i 1}, \ldots, \tau_{i m_{i}}\right\}
\end{gathered}
$$

and

$$
\left|\int_{\tau_{i j}-\delta}^{\tau_{\operatorname{sign}}\left(\tau_{i j}-t_{t}\right)} a_{i}(t) d t\right|=+\infty
$$

for any sufficiently small $\delta>0$ if $\tau_{i j} \notin\left\{a, b ; t_{i}\right\}$.

Then problem (1), (2) is solvable.

THEOREM 1.2. - Let conditions (1.4), (1.6)-(1.8) be fulfilled and let on the domain $\mathfrak{D}_{a b}^{n}$ the inequalities

$$
\begin{aligned}
& f_{i}\left(t, x_{1}, \ldots, x_{n}\right) \operatorname{sign}\left[\left(t-t_{i}\right)\left(x_{i}-x_{0 i}\right)\right] \leqslant \\
& \quad \leqslant-a_{i}(t)\left|x_{i}-x_{0 i}\right|+\sum_{k=1}^{n} a_{i k}(t)\left|x_{k}\right|+h_{i}\left(t, \sum_{k=1}^{n}\left|x_{k}\right|\right) \quad(i=1, \ldots, n)
\end{aligned}
$$


hold, where

$$
A(t)=\left(a_{i k}(t)\right)_{i, k=1}^{n} \in N_{0}\left(a, b ; t_{1}, \ldots, t_{n}\right),
$$

and the functions $h_{i}(t, \varrho) \in K(a, b)(i=1, \ldots, n)$ are nonnegative, nondeoreasing in $\varrho$ and

$$
\lim _{e \rightarrow+\infty} \frac{1}{\varrho} \int_{a}^{b} h_{i}(t, \varrho) d t=0 \quad(i=1, \ldots, n)
$$

Then problem (1), (2) is solvable. If, in addition, $a_{i}(t) \equiv 0(i=1, \ldots, n)$ and the functions $a_{i k}(t) \in L(a, b)$ are nonnegative but does not satisfy conditon (1.10), then there exist functions $f_{i}\left(t, x_{1}, \ldots, x_{n}\right) \in K(a, b)(i=1, \ldots, n)$ satisfying inequalities (1.9) and such that for this funotions problem (1), (2) has no solution.

Coroldary. - Let conditions (1.4), (1.6)-(1.8) hold and let on the domain $\mathfrak{D}_{a b}^{n}$ the inequalities $(1.9)$ be fulfilled, where $h_{i}(t, \varrho)(i=1, \ldots, n)$ are nonnegative and nondecreasing in e functions satisfying conditions (1.11). Then problem (1), (2) is solvable whenever the matrix $A(t)=\left(a_{i k}(t)\right)_{i, k=1}^{n}$ is nonnegative and satisfying one of the following three conditions:

1) $a_{i k}(t) \in L(a, b)(i, k=1, \ldots, n)$ and

$$
\int_{a}^{b}\left\{\sum_{i=1}^{n}\left[\sum_{k=1}^{n} a_{i k}^{q}(t)\right]^{p / q}\right\}^{1 / p} d t<l_{p},
$$

where $p \in(1,+\infty), 1 / p+1 / q=1$

$$
l_{p}=(p-1)^{1 / p}\left(\frac{p}{\pi} \sin \frac{\pi}{p}\right)^{-1}
$$

2) $A(t) \equiv A$ is a constant matrix and all of it proper value modulo is less than $\pi / 2(b-a)$.

3) $a_{i k}(t) \in L^{p_{k}}(a, b), 1 \leqslant p_{k} \leqslant+\infty, 1 / p_{k}+1 / q_{k}=1(i, k=1, \ldots, n)$,

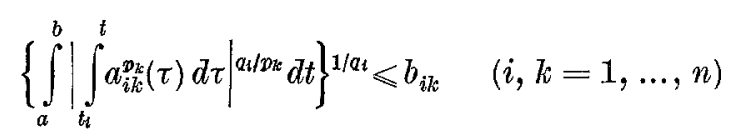

and all proper values of matrix $B=\left(b_{i k}\right)_{i, k=1}^{n}$ modulo are less than one. 
THEOREM 1.3. - Let the conditions (1.4), (1.6) - (1.8) hold and let on the domain $\mathcal{D}_{a b}^{n}$ the following inequalities be fulfilled

$$
\begin{aligned}
& f_{1}\left(t, x_{1}, \ldots, x_{n}\right) \operatorname{sign}\left[\left(t-t_{1}\right)\left(x_{1}-x_{01}\right)\right] \leqslant-a_{1}(t)\left|x_{1}-x_{01}\right|+h_{1}(t) \omega\left(\left|x_{1}-x_{01}\right|\right), \\
& f_{i}\left(t, x_{1}, \ldots, x_{n}\right) \operatorname{sign}\left[\left(t-t_{i}\right)\left(x_{i}-x_{0 i}\right)\right] \leqslant \\
& \quad \leqslant-a_{i}(t)\left|x_{i}-x_{0 i}\right|+h_{i}\left(t, x_{1}, \ldots, x_{i-1}\right) \omega_{i}\left(\left|x_{i}-x_{0 i}\right|\right) \quad(i=2, \ldots, n),
\end{aligned}
$$

where $h_{1}(t) \in L(a, b), h_{i}\left(t, x_{1}, \ldots, x_{i-1}\right) \in K(a, b)(i=2, \ldots, n)$ and functions $\omega_{i}(t)(i=$ $=1, \ldots, n)$ are continuous and positive on the inteval $[0,+\infty)$ and satisty the conditions

$$
\int_{0}^{+\infty} \frac{d t}{\omega_{i}(t)}=+\infty \quad(i=1, \ldots, n) .
$$

Then problem (1), (2) is solvable.

THEOREM 1.4. - If for $\left(t, x_{k 11}, \ldots, x_{k n}\right) \in \mathcal{D}_{a b}^{n}(k=1,2)$ the inequalities

$$
\begin{aligned}
{\left[f_{i}\left(t, x_{11}, \ldots, x_{1 n}\right)-f_{i}\left(t, x_{21}, \ldots, x_{2 n}\right)\right] } & \operatorname{sign}\left[\left(t-t_{i}\right)\left(x_{1 i}-x_{2 i}\right)\right] \leqslant \\
& \leqslant \sum_{k=1}^{n} a_{i k}(t)\left|x_{1 k}-x_{2 k}\right| \quad(i=1=1, \ldots, n)
\end{aligned}
$$

are fulfilled, where

$$
A(t)=\left(a_{i k}(t)\right)_{i, k=1}^{n} \in N_{0}\left(a, b ; t_{1}, \ldots, t_{n}\right)
$$

then problem (1), (2) has at most one solution.

Corollary. - If for $\left(t, x_{k 1}, \ldots, x_{k n}\right) \in \mathbb{D}_{a b}^{n}(k=1,2)$ inequalities (1.17) are fulfilled, where the matrix $A(t)=\left(a_{i k}(t)\right)_{i, k=1}^{n}$ satisfies one of the conditions 1$), 2$ ) and 3 ) of the corollary of theorem 1.2, then problem (1), (2) has at most one solution.

III. - On the sets $N_{0}\left(a, b ; t_{1}, \ldots, t_{n}\right)$ and $N\left(a, b ; t_{1}, \ldots, t_{n}\right)$.

LEMma $1.1\left(^{1}\right)$. - If the function $x(t)$ is absolutely continuous on the segment $[\alpha, \beta], x^{\prime}(t) \in L^{P}(\alpha, \beta)$ and $x\left(t_{0}\right)=0$, where $p \in(1,+\infty)$ and $t_{0} \in[\alpha, \beta]$, then

$$
\int_{a}^{\beta}|x(t)|^{p} d t \leqslant\left(\frac{\beta-\alpha}{l_{p}}\right)^{P} \int_{\alpha}^{\beta}\left|w^{\prime}(t)\right|^{P} d t,
$$

where $l_{P}$ is a number defined by equality (1.13).

(1) See the proof of this lemma in [8]. 
LeinMA 1.2. - If the matrix $A(t)=\left(a_{i k}(t)\right)_{i, k=1}^{n}$ satisfies one of the conditions 1 ), 2) and 3) of corollary of theorem 1.2, then condition (1.10) is satisfied.

Proof. - Let $x_{1}(t), \ldots, x_{n}(t)$ be any solution of system of differential inequalities (1.1), satisfying boundary conditions (2).

Suppose, first of all, that condition 1) of the corollary of theorem 1.2 is fulfilled. Without loss of generality, we can assume, that

$$
\eta=\int_{a}^{b} a_{0}(t) d t>0 \quad \text { where } \quad a_{0}(t)=\left\{\sum_{i=1}^{n}\left[\sum_{i=1}^{n} a_{i \hbar k}^{q}(t)\right]^{p / a}\right\}^{1 / p} .
$$

Put

$$
s=\frac{1}{\eta} \int_{a}^{t} a_{0}(\tau) d \tau, \quad s_{i}=\frac{1}{\eta} \int_{a}^{t_{i}} a_{0}(\tau) d \tau, \quad y_{i}(s)=x_{i}(t) \quad(i=1, \ldots, n)
$$

and apply Hölder's inequality, we find from (1.1) and (2)

$$
y_{i}\left(s_{i}\right)=0 \quad(i=1, \ldots, n) \quad \text { and } \quad \sum_{i=1}^{n}\left|y_{i}^{\prime}(s)\right|^{p} \leqslant \eta^{p} \sum_{i=1}^{n}\left|y_{i}(s)\right|^{p} \quad \text { for } \quad 0 \leqslant s \leqslant 1
$$

Hence, according to lemma 1.1 we have

$$
\sum_{i=1}^{n} \int_{0}^{1}\left|y_{i}^{\prime}(s)\right| d s \leqslant \eta^{P} \sum_{i=1}^{n} \int_{0}^{1}\left|y_{i}(s)\right|^{P} d s \leqslant\left(\frac{\eta}{l_{p}}\right)^{P} \sum_{i=1}^{n} \int_{0}^{1}\left|y_{i}^{\prime}(s)\right|^{P} d s .
$$

By (1.12) and (1.18) it is clear from the obtained inequality, that

$$
\int_{0}^{1}\left|y_{i}^{\prime}(s)\right|^{\infty} d s=0 \quad(i=1, \ldots, m)
$$

and, consequentily,

$$
x_{i}(t) \equiv 0 \quad \text { for } a \leqslant t \leqslant b \quad(i=1, \ldots, n) .
$$

Let us consider now the case when condition 2) of the corollary of theorem 1.2 is satisfied.

According to lemma 1.1 from inequalities (1.1) we find

$$
\begin{aligned}
\int_{a}^{b} x_{i}^{\prime 2}(t) d t \leqslant \sum_{k=1}^{n} a_{i k} \int_{a}^{b}\left|x_{i}^{\prime}(t)\right|\left|x_{k}(t)\right| d t \leqslant \sum_{k=1}^{n} a_{i k}\left[\int_{a}^{b} x_{i}^{\prime 2}(t) d t\right]^{\frac{1}{2}}\left[\int_{a}^{b} x_{k}^{2}(t) d t\right]^{\frac{1}{2}} \leqslant \\
\leqslant \frac{2(b-a)}{\pi} \sum_{k=1}^{n} a_{i k}\left[\int_{a}^{b} x_{i}^{\prime 2}(t) d t\right]\left[\int_{a}^{\frac{1}{2}} x_{k}^{\prime 2}(t) d t\right]^{\frac{1}{2}} \quad(i=1, \ldots, n) .
\end{aligned}
$$


Hence,

$$
\left(E-\frac{2(b-a)}{\pi} A\right) \bar{\varrho} \leqslant \bar{\theta},
$$

where $E$ is the unit matrix,

$$
\bar{\varrho}=\left(\left[\int_{a}^{b} x_{i}^{\prime 2}(t) d t\right]^{\frac{1}{2}}\right)_{i=1}^{n}, \quad \bar{\theta}=(0)_{i=1}^{n} .
$$

Since the spectrum of the matrix $(2(b-a) / \pi) A$ is situated within the unit circle, the matrix $E-(2(b-a) / \pi) A$ has its inverse which is a nonnegative matrix. Hence it is clear from $(1.20)$ that

$$
\bar{\varrho}=\bar{\theta} .
$$

Therefore, by (2) we obtain identities (1.19).

In a similar way we may show, that the system of differential inequalities (1.1) has only the trivial solution satisfying boundary conditions (2) also in the case when condition 3) of the corollary of theorem 1.2 is satisfied.

The lemma is proved.

REMARK. - It should be noted that when $A(t)$ is a constant matrix, then the estimation of proper values suggested in the lemma, cannot be further improved. Indeed, $-\pi / 2$ and $\pi / 2$ are the proper values of the matrix

$$
A=\left(\begin{array}{cc}
0 & 1 \\
\frac{\pi^{2}}{4} & 0
\end{array}\right)
$$

while the system of differential inequalites

$$
\left|x_{1}^{\prime}(t)\right| \leqslant\left|x_{2}(t)\right|, \quad\left|x_{2}^{\prime}(t) \leqslant \frac{\pi^{2}}{4}\right| x_{1}(t) \mid \quad \text { for } 0 \leqslant t \leqslant 1
$$

has the nontrivial solution

$$
x_{1}(t)=\sin \frac{\pi}{2} t, \quad x_{2}(t)=\frac{\pi}{2} \cos \frac{\pi}{2} t
$$

satisfying the boundary conditions

$$
x_{1}(0)=0, \quad x_{2}(1)=0 .
$$

LEMMA 1.3. - Let

$$
g_{i}\left(t, x_{1}, \ldots, x_{n}\right)=\sum_{k=1}^{n} a_{i k}(t)\left|x_{k}\right|+h_{i}\left(t, \sum_{k=1}^{n}\left|x_{k}\right|\right) \quad(i=1, \ldots, n),
$$


where the matrix $A(t)=\left(a_{i k}(t)\right)_{i, k=1}^{n}$ satisfies condition (1.10) and the functions $h_{i}(t, \varrho) \in K(a, b)(i=1, \ldots, n)$ are nonnegative, nondecreasing in $\varrho$ and satisfying conditions (1.11). Then

$$
\left(g_{i}\left(t, x_{1}, \ldots, x_{n}\right)\right)_{i=1}^{n} \in N\left(a, b ; t_{1}, \ldots, t_{n}\right)
$$

Proof. - Assume the contrary. Then we ean find numbers $x_{0 i}(i=1, \ldots, n)$ and sequences of functions absolutely continuous on $[a, b]\left\{x_{i k}(t)\right\}_{k=1}^{+\infty}(i=1, \ldots, n)$, such that

$$
\begin{aligned}
x_{i k}\left(t_{i}\right)=x_{0 i}, \quad\left|x_{i k}^{\prime}(t)\right| \leqslant \sum_{j=1}^{n} & a_{i j}(t)\left|x_{j k}(t)\right|+ \\
& \quad+h_{i}\left(t, \sum_{j=1}^{n}\left|x_{j k}(t)\right|\right) \quad(i=1, \ldots, n ; k=1,2, \ldots)
\end{aligned}
$$

and

$$
\varrho_{k}=\max \left\{\sum_{j=1}^{n}\left|x_{j k}(t)\right|: a \leqslant t \leqslant b\right\} \rightarrow+\infty \quad \text { for } k \rightarrow+\infty
$$

Put

$$
y_{i k}(t)=\frac{x_{i k}(t)}{\varrho_{i k}} \quad(i=1, . ., n ; k=1,2, \ldots)
$$

Then from (1.22) we obtain

$$
\begin{aligned}
y_{i k}\left(t_{i}\right)= & \frac{x_{0 i}}{\varrho_{k}}, \quad\left|y_{i k}(t)-y_{i k}(s)\right| \leqslant \sum_{j=1}^{n} \int_{s}^{t} a_{i j}(\tau)\left|y_{j k}(\tau)\right| d \tau+ \\
& +\frac{1}{\varrho_{k}} \int_{s}^{t} h_{i}\left(\tau, \varrho_{k}\right) d \tau \quad \text { for } a \leqslant s \leqslant t \leqslant b \quad(i=1, \ldots, n ; k=1,2, \ldots) .
\end{aligned}
$$

By (1.11) and (1.23) it follows easily from (1.24) and (1.25) that the sequences $\left\{y_{i \hat{k}}(t)\right\}_{k=1}^{+\infty}(i=1, \ldots, n)$ are uniformly bounded and equicontinuous on $[a, b]$. Consequently, without loss of generality, it can be assumed that these sequences are uniformly convergent.

Putting

$$
y_{i}(t)=\lim _{k \rightarrow+\infty} y_{i k}(t) \quad(i=1, \ldots, n)
$$

from $(1.23)-(1.25)$ we have

$$
y_{i}\left(t_{i}\right)=0 \quad(i=1, \ldots, n), \sup \left\{\sum_{i=1}^{n}\left|y_{i}(t)\right|: a \leqslant t \leqslant b\right\}=1
$$


and

$$
\left|y_{i}(t)-y_{i}(s)\right| \leqslant \sum_{j=1}^{n} \int_{s}^{t} a_{i j}(\tau)\left|y_{i}(\tau)\right| d \tau \quad \text { for } a \leqslant s<t \leqslant b \quad(i=1, \ldots, n) .
$$

Consequently, $y_{1}(t), y_{2}(t), \ldots, y_{n}(t)$ is the nontrivial solution of the system of differential inequalities (1.1), satisfying boundary conditions (2). But this contradicts condition (1.10). The contradiction thus obtained proves the lemma.

LEMMA 1.4. - Let

$$
\begin{aligned}
g_{1}\left(t, x_{1}, . ., x_{n}\right)=h_{1}(t) \omega_{1}\left(x_{1}\right), \quad & g_{i}\left(t, x_{1}, \ldots, x_{n}\right)= \\
& =h_{i}\left(t, x_{1}, \ldots, x_{i-1}\right) \omega_{i}\left(x_{i}\right) \quad(i=2, \ldots, n),
\end{aligned}
$$

where the function $h_{1}(t) \in L(a, b)$ is nonnegative, the functions $h_{i}\left(t, x_{1}, \ldots, x_{n}\right) \in K(a, b)$ $(i=2, \ldots, n)$ are nonnegative and nondecreasing in $x_{1}, \ldots, x_{n}$ and $\omega_{i}(t)(i=1, \ldots, n)$ are functions, continuous and positive on the interval $[0,+\infty)$ satisfying conditions (1.16). Then condition (1.21) is satisfied.

PRoof. - According to (1.16) for any $\eta>0$ we can find positive numbers $c_{i}=c_{i}(\eta)$ $(i=1, \ldots, n)$ such that

$$
\int_{\eta}^{e_{1}} \frac{d t}{\omega_{1}(t)}>\int_{a}^{b} h_{1}(t) d t
$$

and

$$
\int_{\eta}^{c_{t}} \frac{d t}{\omega_{i}(t)}>\int_{a}^{b} h_{i}\left(t, e_{1}, \ldots, e_{i-1}\right) d t \quad(i=2, \ldots, n)
$$

Let $x_{i}(t)(i=1, \ldots, n)$ are functions absolutely continuous on $[a, b]$, satisfying conditions (1.3). Putting

$$
\eta=\sum_{i=1}^{n}\left|x_{0 k}\right| \quad \text { and } \quad \varrho_{i}(t)=\left|x_{i}(t)\right| \quad(i=1, \ldots, n)
$$

according to (1.26) we shall have

$$
\varrho_{1}\left(t_{1}\right) \leqslant \eta, \quad\left|\varrho_{1}^{\prime}(t)\right| \leqslant h_{1}(t) \omega_{1}\left(\varrho_{1}(t)\right) \quad \text { for } a \leqslant t \leqslant b
$$

and

$$
\varrho_{i}\left(t_{i}\right) \leqslant \eta, \quad \varrho_{i}^{\prime}(t) \leqslant h_{i}\left(t, \varrho_{1}(t), \ldots, \varrho_{i-1}(t)\right) \omega_{i}\left(\varrho_{i}(t)\right) \quad \text { for } a \leqslant t \leqslant b \quad(i=2, \ldots, n)
$$


From (1.28) we obtain

$$
\int_{\eta}^{e_{1}(t)} \frac{d s}{\omega_{1}(s)} \leqslant \int_{a}^{b} h_{1}(\tau) d \tau \quad \text { for } a \leqslant t \leqslant b .
$$

Therefore, by (1.27) it follows

$$
\varrho_{1}(t) \leqslant c_{1} \quad \text { for } a \leqslant t \leqslant b \text {. }
$$

Suppose now that for some $i \in\{2, \ldots, n\}$ the inequalities

$$
\varrho_{j}(t) \leqslant c_{j} \quad \text { for } a \leqslant t \leqslant b \quad(j=1, \ldots, i-1)
$$

hold. Then from (1.30) we shall find

$$
\int_{\eta}^{e_{i}(t)} \frac{d s}{\omega_{i}(s)} \leqslant \int_{a}^{b} h_{i}\left(\tau, e_{1}, \ldots, e_{i-1}\right) d \tau \quad \text { for } a \leqslant t \leqslant b .
$$

Hence, by (1.28), it is clear, that

$$
\varrho_{i}(t) \leqslant c_{i} \quad \text { for } a \leqslant t \leqslant b .
$$

Thus we have proved, that

$$
\varrho_{i}(t) \leqslant e_{i} \quad \text { for } a \leqslant t \leqslant b \quad(i=1, \ldots, n) \text {. }
$$

Consequently, inequalities (1.2) hold, where $c_{0}=\sum_{i=1}^{n} c_{i}$. The lemma is proved.

LEMma 1.5. - If condition (1.21) is satisfied, then for arbitrary $x_{0 i} \in(-\infty,+\infty)$ $(k=1, \ldots, n)$ we can find a positive number $c_{0}=c_{0}\left(x_{01}, \ldots, x_{0 n}\right)$, such that the arbitrary functions $x_{i}(t)(i=1, \ldots, n)$ being absolutely continuous on $[a, b]$ and satisfying the conditions

$$
\begin{aligned}
x_{i}\left(t_{i}\right)=x_{0 i}, \quad x_{i}^{\prime}(t) \operatorname{sign}[ & \left.\left(t-t_{i}\right) x_{i}(t)\right] \leqslant \\
& \leqslant g_{i}\left(t,\left|x_{1}(t)\right|, \ldots,\left|x_{n}(t)\right|\right) \quad \text { for } a \leqslant t \leqslant b \quad(i=1, \ldots, n)
\end{aligned}
$$

satisfy also inequalities (1.2). 
Proof. - We choose the constant $c_{0}$ in such a way that arbitrary functions $y_{i}(t)$ $(i=1, \ldots, n)$ being absolutely continuous on $[a, b]$ and satisfying the conditions

$$
y_{i}\left(t_{i}\right)=\left|x_{0 i}\right|, \quad\left|y_{i}^{\prime}(t)\right| \leqslant g_{i}\left(t,\left|y_{1}(t)\right|, \ldots,\left|y_{n}(t)\right|\right) \quad \text { for } a \leqslant t \leqslant b(i=1, \ldots, n)
$$

should satisfy also the inequalities

$$
\left|y_{i}(t)\right| \leqslant c_{0} \quad \text { for } a \leqslant t \leqslant b \quad(i=1, \ldots, n) .
$$

Let $x_{i}(t)(i=1, \ldots, n)$ be some functions, absolutely continuous on $[a, b]$ and satisfying conditions (1.31). Assume

$$
\begin{aligned}
& \varrho_{0}=\max \left\{\sum_{i=1}^{n}\left|x_{i}(t)\right|: a \leqslant t \leqslant b\right\}, \\
& \chi(s)= \begin{cases}1 & \text { for }|s| \leqslant \varrho_{0}+1 \\
2-\frac{|s|}{\varrho_{0}+1} & \text { for } \varrho_{0}+1<|s|<2 \varrho_{0}+2 \\
0 & \text { for }|s| \geqslant 2 \varrho_{0}+2\end{cases}
\end{aligned}
$$

and

$$
\begin{array}{r}
\omega_{i}(t, s)=\chi(s) g_{i}\left(t,\left|x_{1}(t)\right|, \ldots,\left|x_{i-1}(t)\right|, s,\left|x_{i+1}(t)\right|, \ldots,\left|x_{n}(t)\right|\right) \operatorname{sign}\left(t-t_{i}\right) \\
(i=1, \ldots, n) .
\end{array}
$$

It is clear that on the domain $\mathfrak{D}_{a b}^{1}$ the inequalities

$$
\left|\omega_{i}(t, s)\right| \leqslant \sup \left\{\left|g_{i}\left(t, x_{1}, \ldots, x_{n}\right)\right|: \sum_{k=1}^{n}\left|x_{k}\right| \leqslant 2 \varrho_{0}+2\right\} \in L(a, b) \quad(i=1, \ldots, n)
$$

hold. Therefore for any $i \in\{1, \ldots, n\}$ the problem

$$
\frac{d y}{d t}=\omega_{i}(t, y), \quad y\left(t_{i}\right)=\left|x_{0 i}\right|
$$

has the upper solution $y_{i}(t)$ defined on $[a, b]$. On the other hand, by $(1.31),(1.34)$ and $(1.35)$

$$
\left|x_{i}\left(t_{i}\right)\right|=\left|x_{0 i}\right|, \quad\left[\frac{d\left|x_{i}(t)\right|}{d t}-\omega_{i}\left(t,\left|x_{i}(t)\right|\right)\right] \operatorname{sign}\left(t-t_{i}\right) \leqslant 0 \quad \text { for } a \leqslant t \leqslant b \quad(i=1, \ldots, n)
$$

Therefore it follows that

$$
\left|x_{i}(t)\right| \leqslant y_{i}(t) \quad \text { for } a \leqslant t \leqslant b(i=1, \ldots, n)\left({ }^{2}\right) .
$$

(2) See [4], comparison lemma.

11 - Annali di Matematica 
It is easily seen that $y_{i}(t)(i=1, \ldots, n)$ satisfy conditions (1.32). Consequently, as stated above, they satisfy inequalities (1.33) too. Hence inequalities (1.2) hold. The lemma is proved.

In an analogous way the following lemma can be proved.

LEMMa 1.6. - If condition (1.10) holds, then the system of differential inequalities

$$
x_{i}^{\prime}(t) \operatorname{sing}\left[\left(t-t_{i}\right) x_{i}(t)\right] \leqslant \sum_{k=1}^{n} a_{i k}(t)\left|x_{k}(t)\right| \quad \text { for } a<t<b(i=1, \ldots, n)
$$

has only the trivial soution, satisfying the boundary conditions (2).

IV. - The proof of existence and uniqueness theorems.

Below we shall need the following simple

LEMMA 1.7. - Let a function $x(t)$ be bounded on $(\alpha, \beta)$ and absolutely continuous on every segment contained within this interval. Besides, we can find the function $h(t) \in L(\alpha, \beta)$, such that either

$$
x^{\prime}(t) \geqslant h(t) \quad \text { for } \alpha<t<\beta,
$$

or

$$
x^{\prime}(t) \leqslant h(t) \quad \text { for } \alpha<t<\beta .
$$

Then there exist the finite limits

$$
\lim _{t \rightarrow \infty} x(t), \quad \lim _{t \rightarrow \beta} x(t)
$$

and if we take them for $x(\alpha)$ and $x(\beta)$ correspondingly, then $x(t)$ will be absolutely continuous on $[\alpha, \beta]$.

Proof. Let $t_{0} \in(\alpha, \beta)$. The function

$$
y(t)=x(t)-\int_{t_{0}}^{t} h(\tau) d \tau
$$

is monotonic and bounded on $(\alpha, \beta)$. Hence $y^{\prime}(t) \in L(\alpha, \beta)$. Consequently, $x^{\prime}(t) \in$ $\in L(\alpha, \beta)$ and

$$
x(t)=x\left(t_{0}\right)+\int_{t_{0}}^{t} x^{\prime}(\tau) d \tau \quad \text { for } \alpha<t<\beta
$$

which was to be proved. 
PROOF OF THEOREM 1.1. - Let $e_{0}$ be a positive number chosen according to lemma 1.5 for $x_{01}, \ldots, x_{0 n}$. Putting

$$
\begin{aligned}
& \Delta_{i j}(\eta)= \begin{cases}\left(\tau_{i j}-\eta, \tau_{i j}+\eta\right) \cap(a, b) & \text { for }\left|\tau_{i j}\right|<+\infty \\
\left(-\infty,-\frac{1}{\eta}\right) & \text { for } \tau_{i j}=-\infty \\
\left(\frac{1}{\eta},+\infty\right) & \text { for } \tau_{i j}=+\infty\end{cases} \\
& \Delta_{i}(\eta)=\bigcup_{j=1}^{m_{t}} \Delta_{i j}(\eta) \quad(i=1, \ldots, n), \\
& c_{1}=n c_{0}+\sum_{i=1}^{n}\left|x_{0 i}\right|, \quad \chi(s)= \begin{cases}1 & \text { for } 0 \leqslant s \leqslant c_{1} \\
2-s / c_{1} & \text { for } c_{1}<s<2 c_{1}, \\
0 & \text { for } s \geqslant 2 e_{1}\end{cases} \\
& f_{i k}\left(t, x_{1}, \ldots, x_{n}\right)= \begin{cases}f_{i}\left(t, x_{1}, \ldots, x_{n}\right) & \text { for } t \notin \Delta_{i}\left(\frac{1}{k}\right) \\
0 & \text { for } t \in \Delta_{i}\left(\frac{1}{k}\right),\end{cases} \\
& f_{i k}^{*}\left(t, x_{1}, \ldots, x_{n}\right)=\chi\left(\sum_{j=1}^{n}\left|x_{j}\right|\right) f_{i k}\left(t, x_{1}, \ldots, x_{n}\right) \quad(i=1, \ldots, n) .
\end{aligned}
$$

From (1.3) and (1.36)-(1.38) it is clear that for arbitrarily chosen natural number $k$

$$
f_{i k}^{*}\left(t, x_{1}, \ldots, x_{n}\right) \in K(a, b) \quad(i=1, \ldots, n)
$$

and on the domain $\mathfrak{D}_{a b}^{n}$ the inequalities

$$
\left|f_{i k}^{*}\left(t, x_{1}, \ldots, x_{n}\right)\right| \leqslant f_{i k}^{*}(t) \quad(i=1, \ldots, n)
$$

hold, where $f_{i k}^{*}(t) \in L(a, b)(i=1, \ldots, n)$. Hence, applying Shauder's principle [15] it is easy to see that the system of integral equations

$$
x_{i}(t)=\int_{t_{i}}^{t} f_{i k i}^{*}\left(\tau, x_{1}(\tau), \ldots, x_{n}(\tau)\right) d \tau \quad(i=1, \ldots, n)
$$

has the solution $x_{1 k}(t), \ldots, x_{n k}(t)$ continuous on $[a, b]$.

Putting

$$
y_{i k}(t)=x_{i k}(t)-x_{0 i} \quad(i=1, \ldots, n)
$$


by (1.5) and (1.36)-(1.38) we shall have

$$
\begin{array}{r}
y_{i k}\left(t_{i}\right)=-x_{0 i}, \quad y_{i k}^{\prime}(t) \operatorname{sign}\left[\left(t-t_{i}\right) y_{i k}(t)\right] \leqslant g_{i}\left(t,\left|y_{1 k}(t)\right|, \ldots,\left|y_{n k}(t)\right|\right) \\
\text { for } a \leqslant t \leqslant b(i=1, \ldots, n) .
\end{array}
$$

According to lemma 1.5 it follows from this, that

$$
\left|y_{i k}(t)\right| \leqslant c_{0} \quad \text { for } a \leqslant t \leqslant b(i=1, \ldots, n ; k=1,2, \ldots) .
$$

Consequently,

$$
\left|x_{i k}(t)-x_{0 i}\right| \leqslant c_{0} \quad \text { for } a \leqslant t \leqslant b(i=1, \ldots, n ; k=1,2, \ldots)
$$

and

$$
\left|x_{i k}(t)\right| \leqslant\left|x_{0 i}\right|+\left|\int_{t_{i}}^{t} g(\tau) d \tau\right| \quad \text { for } a \leqslant t \leqslant b \quad(i=1, \ldots, n ; k=1,2, \ldots)
$$

where

$$
g(t)=\sup \left\{\sum_{i=1}^{n}\left|g_{i}\left(t, x_{1}, \ldots, x_{n}\right)\right|:\left|x_{k}\right| \leqslant c_{0}(k=1, \ldots, n)\right\} \in L(a, b)
$$

From $(1.36)-(1.39)$ it is clear that $x_{1 k}(t), \ldots, x_{n k}(t)$ is the solution of the system

$$
\frac{d x_{i}}{d t}=f_{i k}\left(t, x_{1}, \ldots, x_{n}\right) \quad(i=1, \ldots, n)
$$

satisfying boundary conditions (2).

According to (1.3), (1.4), (1.36) - (1.39) and (1.41)

$$
\begin{gathered}
x_{i k}^{\prime}(t)=0 \quad \text { for } t \in \Delta_{i}\left(\frac{1}{k}\right), \quad x_{i k}^{\prime}(t) \operatorname{sign}\left[\left(t-t_{i}\right)\left(x_{i k}(t)-x_{0 i}\right)\right] \leqslant \\
\leqslant-a_{i}(t)\left|x_{i k}(t)-x_{0 i}\right|+g(t) \quad \text { for } t \in\lfloor a, b] \backslash \Delta_{i}\left(\frac{1}{k}\right) \quad(i=1, \ldots, n ; k=1,2, \ldots), \\
\left|x_{i k}^{\prime}(t)\right| \leqslant f_{i}(t) \quad \text { for } t \in[a, b](i=1, \ldots, n),
\end{gathered}
$$

where

$$
f_{i}(t)=\sup \left\{\left|f_{i}\left(t, x_{1}, \ldots, x_{n}\right)\right|:\left|x_{k}\right| \leqslant e_{1}: k=1, \ldots, n\right\} \in L\left(a, b, \tau_{i 1}, \ldots, \tau_{i m i}\right)
$$

Let us choose $\eta>0$ so small that whenever $\tau_{i j} \notin\left\{a, b ; t_{i}\right\}$ we shall have

$$
\left[\tau_{i j}-\eta, \tau_{i j}+\eta\right] \subset(a, b) \quad \text { and } \quad \tau_{i l} \notin\left[\tau_{i j}-\eta, \tau_{i j}+\eta\right] \quad \text { for } l \neq j \text {. }
$$


If $\tau_{i j}>t_{i}$ then we suppose

$$
\begin{gathered}
\sigma_{i j}(t)=\left[c_{0}+\int_{\tau_{i j}-\eta} g(\tau) \exp \left(\int_{\tau_{i j}-\eta}^{\tau} a_{i}(s) d s\right) d \tau\right] \exp \left[-\int_{\tau_{i j}-\eta}^{t} a_{i}(\tau) d \tau\right], \\
\sigma_{i j k}(t)=\left\{\begin{array}{cc}
\sigma_{i j}(t)+\sigma_{i j}\left(\tau_{i j}-\frac{1}{k}\right) & \text { for } t<\tau_{i j}-\frac{1}{k} \\
0 & \text { for } t \in \Delta_{i j}\left(\frac{1}{k}\right) . \\
\sigma_{i j}\left(\tau_{i j}-\frac{1}{k}\right)+\int_{\tau_{i j}}^{t} g_{i}(\tau) d \tau & \text { for } t>\tau_{i j}+\frac{1}{k}
\end{array}\right.
\end{gathered}
$$

And if $\tau_{i j}<t_{i}$, then

$$
\begin{gathered}
\sigma_{i j}(t)=\left[c_{0}+\int_{i}^{\tau_{i j}+\eta} g(\tau) \exp \left(\int_{\tau}^{\tau_{i j}+\eta} a_{i}(s) d s\right) d \tau\right] \exp \left[-\int_{i}^{\tau_{i j}+\eta} a_{i}(\tau) d \tau\right], \\
\sigma_{i j k}(t)=\left\{\begin{array}{cc}
\sigma_{i j}(t)+\sigma_{i j}\left(\tau_{i j}+\frac{1}{k}\right) & \text { for } t>\tau_{i j}+\frac{1}{k} \\
0 & \text { for } t \in \Delta_{i j}\left(\frac{1}{k}\right) . \\
\sigma_{i j}\left(\tau_{i j}+\frac{1}{k}\right)+\int_{t}^{\tau_{i j}} g_{i}(\tau) d \tau & \text { for } t<\tau_{i j}-\frac{1}{k}
\end{array}\right.
\end{gathered}
$$

Let

$$
\gamma_{i j}(t)=\left|\int_{t_{i}}^{t} g_{i}(\tau) d \tau\right| \quad \text { for } \tau_{i j}=t_{i}
$$

and

$$
\gamma_{i j}(t)=\sup \left\{\sigma_{i j k}(t): k=1,2, \ldots\right\} \quad \text { for } \tau_{i j} \neq t_{i}
$$

Then by (1.8) and (1.41) we have

$$
\lim _{i \rightarrow \tau_{t_{j}}} \gamma_{i j}(t)=0 \quad(i, j=1, \ldots, n) .
$$

According to (1.7), (1.40) and (1.42) if $a<\tau_{i j}<b$ or $\tau_{i j}=t_{i}$, then

$$
\left|x_{i k}(t)-x_{i k}\left(\tau_{i j}\right)\right| \leqslant \gamma_{i j}(t) \quad \text { for } t \in \Delta_{i j}(\eta)
$$


From (1.39) and (1.43)-(1.46) it follows easily, that sequences $\left\{x_{i k}(t)\right\}_{k=1}^{+\infty}$ are uniformly bounded and equicontinuous on every segment contained in the interval $(a, b)$. Consequently, by the Arzela-Ascoli Lemma, without loss of generality, we can suppose, that they are uniformly convergent on every segment contained in the interval $(a, b)$.

Taking into account $(1.39),(1.45)$ and (1.46) it can be easily shown, that

$$
x_{i}(t)=\lim _{k \rightarrow+\infty} x_{i k}(t) \quad(i=1, \ldots, n)
$$

satisfy boundary conditions (2) and

$$
\left|x_{i}(t)-x_{\theta i}\right| \leqslant c_{0} \quad \text { for } a \leqslant t \leqslant b(i=1, \ldots, n)
$$

Let $[\alpha, \beta] \subset(a, b)$ be an arbitrary segment, which contains no points $\tau_{i j}(j=1$, $\left.\ldots, m_{i} ; i=1, \ldots, n\right)$. By (1.38), if we proceed to the limit in equalities

$$
x_{i k}(t)=x_{i k}(\alpha)+\int_{\alpha}^{t} f_{i k}\left(\tau, x_{1 k}(\tau), \ldots, x_{n k}(\tau)\right) d \tau \quad(i=1, \ldots, n)
$$

for $k \rightarrow+\infty$, we obtain

$$
x_{i}(t)=x_{i}(\alpha)+\int_{\alpha}^{t} f_{i}\left(\tau, x_{1}(\tau), \ldots, x_{n}(\tau)\right) d \tau \quad(i=1, \ldots, n) .
$$

Consequently, $x_{i}(t)(i=1, \ldots, n)$ satisfy system (1) almost everywhere on $(a, b)$ and they are absolutely continuous on every segment $[\alpha, \beta] \subset(a, b)$ which contains no points $\tau_{i j}\left(j=1, \ldots, m_{i} ; i=1, \ldots, n\right)$.

By (1.5), (1.41) and (1.46)

$$
\frac{d\left|x_{i}(t)-x_{0 i}\right|}{d t} \operatorname{sign}\left(t-t_{i}\right) \leqslant g_{i}(t) \quad \text { for } a<t<b(i=1, \ldots, n) .
$$

Hence, using lemma 1.7, we can conclude, that $x_{i}(t)(i=1, \ldots, n)$ are absolutely continuous on $(a, b)$. Consequently, $x_{i}(t)(i=1, \ldots, n)$ is a solution of problem (1), (2). Thus the theorem is proved.

THE PROOF OF THEOREM 1.2. - By (1.9) it is obvious that on the domain $\mathfrak{D}_{a b}^{n}$ the inequalities (1.5) hold, where

where

$$
g_{i}\left(t, x_{1}, \ldots, x_{n}\right)=\sum_{k=1}^{n} a_{i \hbar}(t)\left|x_{k}\right|+h_{i}^{*}\left(t, \sum_{k=1}^{n}\left|x_{k}\right|\right) \quad(i=1, \ldots, n)
$$

$$
h_{i}^{*}(t, \varrho)=\sum_{k=1}^{n} a_{i k}(t)\left|x_{0 k}\right|+h_{i}\left(t, \sum_{k=1}^{n}\left|x_{0 k}\right|+\varrho\right) \quad(i=1, \ldots, n) .
$$


Since $h_{i}^{*}(t, \varrho)(i=1, \ldots, n)$ satisfy the same conditions as $h_{i}(t, \varrho)(i=1, \ldots, n)$, by lemma 1.3 the condition (1.21) holds. Thus solvability of problem (1), (2) is proved.

We proceed to the proof of the second part of the theorem. Let the functions $a_{i k}(t) \in L(a, b)(i, k=1, \ldots, n)$ be nonnegative and satisfy condition (1.10). Then the system of differential inequalities $(1.1)$ has the nontrivial solution $x_{i}(t)(i=1, \ldots, n)$ satisfying the boundary conditions (2). Putting

and

$$
\eta_{i}(t)=\sum_{k=1}^{n} a_{i k}(t)\left|x_{k}(t)\right| \quad(i=1, \ldots, n)
$$

$$
b_{i n}(t)=\left\{\begin{array}{ll}
\frac{x_{i}^{t}(t)}{\eta_{i}(t)} a_{i k}(t) \operatorname{sign} x_{k}(t) & \text { for } \eta_{i}(t) \neq 0 \\
0 & \text { for } \eta_{i}(t)=0
\end{array} \quad(i, k=1, \ldots, n) .\right.
$$

It is clear that $x_{i}(t)(i=1, \ldots, n)$ is a solution of the system

$$
\frac{d x_{i}}{d t}=\sum_{k=1}^{n} b_{i k}(t) x_{k} \quad(i=1, \ldots, n) .
$$

Since problem (1.48), (2) has a nontrivial solution, we can find functions $b_{i}(t) \in$ $\in L(a, b)(i=1, \ldots, n)$, such that the system of differential equations

$$
\frac{d x_{i}}{d t}=\sum_{k=1}^{n} b_{i k}(t) x_{k}+b_{i}(t) \quad(i=1, \ldots, n)
$$

has no solution, satisfying the boundary conditions (2). Taking into account, that the functions

$$
f_{i}\left(t, x_{1}, \ldots, x_{n}\right)=\sum_{k=1}^{n} b_{i k i}(t) x_{k}+b_{i}(t)
$$

satisfy the inequalities (1.9), where $a_{i}(t) \equiv 0, x_{0 i}=0$ and $h_{i}(t, \varrho) \equiv\left|b_{i}(t)\right|(i=1, \ldots, n)$. Thus the validity of theorem 1.2 is clear.

If we take into account lemma 1.2 , it can be easily verified that the corollary of this theorem is valid.

We obtian theorem 1.3 from theorem 1.1 using lemma 1.4 .

As regards theorem 1.4 and its corollary, they follow immediately from the lemmas 1.2 and 1.6.

\section{2. - The theorems of existence and uniqueness for equation of $n$-th order.}

Let us consider the problem

$$
\begin{aligned}
& u^{(n)}=f\left(t, u, u^{\prime}, \ldots, u^{(n-1)}\right), \\
& u^{(i-1)}\left(t_{i}\right)=0 \quad(i=1, \ldots, n),
\end{aligned}
$$


where $a \leqslant t_{i} \leqslant b(i=1, \ldots, n)$. Under the solution of this problem we shall mean a function $u(t)$, which along with its derivatives to order $n-1$ inclusive, is absolutely continuous of the interval $[a, b]$ and satisfies there almost everywhere equation (2.1) and boundary conditions $(2.2)$.

Theorem 2.1. - Let

$$
f\left(t, x_{1}, \ldots, x_{n}\right) \in K\left(a, b ; t_{n}\right)
$$

and on the domain $D_{a b}^{n}$ the inequality

$$
f\left(t, x_{1}, \ldots, x_{n}\right) \operatorname{sign}\left[\left(t-t_{n}\right) x_{n}\right] \leqslant \sum_{k=1}^{n} a_{k}(t)\left|x_{k}\right|+h\left(t, \sum_{k=1}^{n}\left|x_{k}\right|\right)
$$

hold, where the function $h(t, \varrho) \in K(a, b)$ is nonnegative, nondecreasing in $\varrho$ and

$$
\lim _{\varrho \rightarrow+\infty} \frac{1}{\varrho} \int_{a}^{b} h(t, \varrho) d t=0
$$

Then the problem (2.1), (2.2) is solvable if one of the following two conditions is satisfied:

1) $a_{k}(t) \equiv a_{k}(k=1, \ldots, n)$ are nonnegative constants satisfying the inequality

$$
\sum_{k=1}^{n}\left[\frac{2(b-a)}{\pi}\right]^{n+1-k} a_{k}<1
$$

2) The functions $a_{k}(t) \in L^{q}(a, b)(k=1, \ldots, n-1)$ and $a_{n}(t) \in L(a, b)$ are nonnegative and

$$
\sum_{k=1}^{n-1}\left(\frac{b-a}{l_{p}}\right)^{n-k}\left\{\int_{a}^{b}\left|\int_{t_{n}}^{t} a_{k}^{q}(\tau) \exp \left(q\left|\int_{\tau}^{t} a_{n}(s) d s\right|\right) d \tau\right|^{p / \alpha} d t\right\}^{1 / \nu}<1
$$

where $1<q<+\infty, 1 / p+1 / q=1$, and $l_{p}$ is a number defined by equality (1.12).

Proof. - According to theorem 1.2 in order to prove the theorem 2.1 it suffices to show, that the system of differential inequalities

$$
\left|x_{k}^{\prime}(t)\right| \leqslant\left|x_{k+1}(t)\right| \quad(k=1, \ldots, n-1), \quad\left|x_{n}^{\prime}(t)\right| \leqslant \sum_{i=1}^{n} a_{i}(t)\left|x_{i}(t)\right| \text { for } a<t<b
$$

has only the trivial solution, satisfying boundary conditions (2). 
Let $x_{1}(t), \ldots, x_{n}(t)$ be an arbitrary solution of system (2.5) with boundary conditions (2).

At first we consider the case 1 ).

According to lemma 1.1

$$
\left[\int_{b}^{b} x_{k}^{2}(t) d t\right]^{k} \leqslant \frac{2(b-a)}{\pi}\left[\int_{a}^{b} x_{k}^{\prime 2}(t) d t\right]^{\frac{1}{2}} \quad(k=1, \ldots, n) .
$$

Consequently from (2.5) we obtain

$$
\left[\int_{a}^{b} x_{i}^{2}(t) d t\right]^{\frac{1}{2}} \leqslant\left[\frac{2(b-a)}{\pi}\right]^{n+1-i}\left[\int_{a}^{b} x_{n}^{\prime 2}(t) d t\right]^{t} \quad(i=1, \ldots, n)
$$

and

$$
\left[\int_{a}^{b} x_{n}^{\prime 2}(t) d t\right]^{\frac{1}{2}} \leqslant \sum_{k=1}^{n} a_{\bar{k}}\left[\int_{a}^{b} x_{k}^{2}(t) d t\right]^{\frac{1}{2}} \leqslant\left\{\sum_{k=1}^{n}\left[\frac{2(b-a)}{\pi}\right]^{n+1-k} a_{k k}\right\}\left[\int_{a}^{b} x_{n}^{\prime 2}(t) d t\right]^{\frac{1}{2}} .
$$

From these inequalities by $(2.3)$ it follows, that $x_{i}(t) \equiv 0$ for $a \leqslant t \leqslant b(i=1, \ldots, n)$.

Let us proceed now to the case 2 ).

Using (2) from (2.5) it follows easily, that

$$
\begin{aligned}
\left|x_{n}(t)\right| \leqslant \sum_{k=1}^{n-1} \mid \int_{t_{n}}^{t} \exp \left(\left|\int_{\tau}^{t} a_{n}(s) d s\right|\right) & a_{k}(\tau)\left|x_{k}(\tau)\right| d \tau \mid \leqslant \\
& \leqslant \sum_{k=1}^{n-1}\left|\int_{t_{n}}^{t} \exp \left(q \mid \int_{\tau}^{t} a_{n}(s) d s\right) a_{k}^{q}(\tau) d \tau\right|^{1 / q}\left[\int_{a}^{b}\left|x_{k}(\tau)\right|^{p} d \tau\right]^{1 / p}
\end{aligned}
$$

and

$$
\left[\int_{a}^{b}\left|x_{n}(\tau)\right|^{p} d \tau\right]^{1 / p} \leqslant \sum_{k=1}^{n-1}\left\{\int_{a}^{b}\left|\int_{t_{n}}^{t} a_{k}^{q}(\tau) \exp \left(q\left|\int_{\tau}^{t} a_{n}(s) d s\right|\right) d \tau\right|^{p / q} d t\right\}^{1 / p}\left[\int_{a}^{b}\left|x_{k}(\tau)\right|^{p} d \tau\right]^{1 / p}
$$

Aceording to lemma 1.1 and the inequalities $(2.5)$, we have

$$
\left[\int_{a}^{b}\left|x_{k}(\tau)\right|^{p} d \tau\right]^{1 / p} \leqslant\left(\frac{b-a}{l_{p}}\right)^{n-k}\left[\int_{a}^{b}\left|x_{n}(\tau)\right|^{p} d \tau\right]^{1 / p} \quad(k=1, \ldots, n-1) .
$$

Taking advantage of condition (2.4), from (2.6) and (2.7) we obtain that $x_{l 4}(t) \equiv 0$ for $a \leqslant t \leqslant b(k=1, \ldots, n)$. Thus the theorem is proved. 
THEOREM 2.2. - Let

$$
\begin{array}{r}
\sigma_{n}(t)=1, \quad \sigma_{i}(t)=\left|\int_{t_{\mathfrak{1}}}^{t} \sigma_{i+1}(\tau) d \tau\right| \quad(i=n-1, \ldots, 1), \\
f\left(t, \sigma_{1}(t) x_{1}, \ldots, \sigma_{n}(t) x_{n}\right) \in K\left(a, b ; t_{n}\right)
\end{array}
$$

and on the domain $D_{a b}^{n}$ the inequality

$$
f\left(t, x_{1}, \ldots, x_{n}\right) \operatorname{sign}\left[\left(t-t_{n}\right) x_{n}\right] \leqslant a_{0}(t)+\sum_{k=1}^{n} a_{k}(t)\left|x_{k}\right|
$$

holds, where the functions $a_{k}(t)(k=0, \ldots, n)$ are nonnegative,

$$
a_{0}(t) \in L(a, b), \sigma_{k}(t) a_{k}(t) \in L(a, b) \quad(k=1, \ldots, n)
$$

and

$$
\sum_{k=1}^{n} \int_{a}^{b} a_{k}(t) \sigma_{k}(t) d t<1
$$

Then the problem (2.1), (2.2) is solvable.

Proof. - We choose the positive number $\varrho_{0}$ in such a way that

$$
\frac{n}{\varrho_{0}} \int_{a}^{b} a_{0}(t) d t+\sum_{k=1}^{n} \int_{a}^{b} a_{k}(t) \sigma_{k}(t) d t<1 .
$$

Putting

$$
\chi(s)= \begin{cases}1 & \text { for } 0 \leqslant s \leqslant \varrho_{0} \\ 2-s / \varrho_{0} & \text { for } \varrho_{0}<s<2 \varrho_{0} \\ 0 & \text { for } s \geqslant 2 \varrho_{0}\end{cases}
$$

and

$$
f^{*}\left(t, x_{1}, \ldots, x_{n}\right)=\chi\left(\sum_{k=1}^{n}\left|x_{k}\right| / \sigma_{k}(t)\right) f\left(t, x_{1}, \ldots, x_{n}\right) .
$$

It is obvious that

$$
f^{*}\left(t, x_{1}, \ldots, x_{n}\right) \in K\left(a, b ; t_{n}\right)
$$

and on the domain $\mathfrak{D}_{a b}^{n}$ the inequality

$$
f^{*}\left(t, x_{1}, \ldots, x_{n}\right) \operatorname{sign}\left[\left(t-t_{n}\right) x_{n}\right] \leqslant h(t)
$$


holds, where

$$
h(t)=a_{0}(t)+2 \varrho_{0} \sum_{k=1}^{n} a_{k}(t) \sigma_{k}(t) \in I(a, b) .
$$

Hence, by theorem 2.1, the equation

$$
u^{(n)}=f^{*}\left(t, u, \ldots, u^{(n-1)}\right)
$$

has a solution $u(t)$ satisfying boundary conditions (2.2).

Putting

$$
\varrho=\max \left\{\left|u^{(n-1)}(t)\right|: a \leqslant t \leqslant b\right\}
$$

by (2.8) and (2.9) we obtain

$$
\left|u^{(k-1)}(t)\right| \leqslant \varrho \sigma_{k}(t) \quad \text { for } a \leqslant t \leqslant b \quad(k=1, \ldots, n)
$$

and

$$
\begin{aligned}
&\left|u^{(n-1)}(t)\right| \leqslant\left|\int_{t_{n}}^{t}\left[a_{0}(\tau)+\sum_{k=1}^{n} a_{k}(\tau)\left|u^{(k-1)}(\tau)\right|\right] d \tau\right| \leqslant \\
& \qquad \int_{a}^{b} a_{0}(\tau) d \tau+\varrho \sum_{k=1}^{n} \int_{a}^{b} a_{k}(\tau) \sigma_{k}(\tau) d \tau \quad \text { for } a \leqslant t \leqslant b .
\end{aligned}
$$

Consequently,

$$
\varrho \leqslant \int_{a}^{b} a_{0}(\tau) d \tau+\varrho \sum_{k=1}^{n} \int_{a}^{b} a_{z}(\tau) \sigma_{k}(\tau) d \tau
$$

Therefore, by (2.11), we obtain that

$$
\varrho \leqslant \frac{\varrho_{0}}{n}
$$

Thus we have proved that

$$
\left|u^{(k-1)}(t)\right| \leqslant \frac{\varrho_{0}}{n} \sigma_{k}(t) \quad \text { for } a \leqslant t \leqslant b \quad(k=1, \ldots, n) .
$$

Using these inequalities from definition of $f^{*}\left(t, x_{1}, \ldots, x_{n}\right)$ it is clear, that $u(t)$ is the solution of equation (2.1). Thus the theorem is proved.

As it was stated above when proving theorem 2.1, the system of differential inequalities (2.5) has only the trivial solution, satisfying boundary conditions (2). Due to this from theorem 1.4 we obtain the following 
THEOREM 2.3. - Let for $\left(t, x_{i 1}, \ldots, x_{i n}\right) \in \mathfrak{D}_{a b}^{n}(i=1,2)$ the inequality

$$
\left[f\left(t, x_{11}, \ldots, x_{1 n}\right)-f\left(t, x_{21}, \ldots, x_{2 n}\right)\right] \operatorname{sign}\left[\left(t-t_{n}\right)\left(x_{1 n}-x_{2 n}\right)\right] \leqslant \sum_{k=1}^{n} a_{k(k}(t)\left|x_{1 k}-x_{2 k}\right|
$$

hold, where the functions $a_{k}(t)(k=1, \ldots, n)$ satisfy either condition 1$)$, or condition 2$)$ of theorem 2.1. Then problem (2.1), (2.2) has at most one solution.

THEOREM 2.4. - Let for $\left(t, x_{i 1}, \ldots, x_{i n}\right) \in \mathfrak{D}_{a b}^{n}(i=1,2)$ the inequality (2.14) hold, where $a_{k}(t)(k=1, \ldots, n)$ are functions satisfying the conditions of theorem 2.2. Then problem (2.1), (2.2) has at most one solution.

Proof. - Let $u_{1}(t)$ and $u_{2}(t)$ be solutions of problem (2.1), (2.2). Putting

$$
u(t)=u_{1}(t)-u_{2}(t)
$$

According to $(2.12)$

$$
\frac{d\left|u^{(n-1)}(t)\right|}{d t} \operatorname{sign}\left(t-t_{n}\right) \leqslant \sum_{k=1}^{n} a_{k}(t)\left|u^{(k-1)}(t)\right| \quad \text { for } a \leqslant t \leqslant b .
$$

Therefore, by (2.12) and (2.13) we find

$$
\varrho \leqslant \varrho \sum_{k=1}^{n} \int_{a}^{b} a_{k}(t) \sigma_{k}(t) d t
$$

From the obtained inequality, by (2.10), it follows, that $\varrho=0$. Consequently, $u_{1}(t)=u_{2}(t)$ for $a \leqslant t \leqslant b$. The theorem is proved.

\section{3. - The case of violation of uniqueness.}

In this section we establish criteria for the existence of the infinite set of the solutions of the problems (1), (2) and (2.1), (2.2).

THEOREM 3.1. - Let

$$
f_{i}\left(t, x_{1}, \ldots, x_{n}\right) \in K\left(a, b ; t_{i}\right) \quad(i=1, \ldots, n)
$$

and let for arbitrary $i \in\{1, \ldots, n\}$ one of the following three conditions be satisfied:

1) $f_{i}\left(t, x_{1}, \ldots, x_{n}\right) \operatorname{sign}\left[\left(t-t_{i}\right) x_{i}\right] \leqslant \varphi_{i}(t)\left|x_{i}\right|+\psi_{i}(t)$, where $\varphi_{i}(t) \in L(a, b)$ and $\psi_{i}(t) \in L(a, b)$; 
2) $f_{i}\left(t, x_{1}, \ldots, x_{n}\right) \operatorname{sign} x_{i} \geqslant \varphi_{i}(t)\left|x_{i}\right|-\psi_{i}(t)$,

where the functions $\varphi_{i}(t) \in L\left(a, b ; t_{i}\right)$ and $\psi_{i}(t) \in L(a, b)$ are nonnegative, $a \leqslant t_{i}<b$ and

$$
\int_{i_{i}}^{b} \varphi_{i}(\tau) d \tau=+\infty
$$

3) $f_{i}\left(t, x_{1}, \ldots, x_{n}\right) \operatorname{sign} x_{i} \leqslant-\varphi_{i}(t)\left|x_{i}\right|+\psi_{i}(t)$,

where the functions $\varphi_{i}(t) \in L\left(a, b ; t_{i}\right)$ and $\psi_{i}(t) \in L(a, b)$ are nonnegative, $a<t_{i} \leqslant b$ and

$$
\int_{a}^{t_{1}} \varphi_{i}(\tau) d \tau=+\infty
$$

If, furthermore, for some $i \in\{1, \ldots, n\}$ either condition 2), or condition 3) is necessarily satisfied, then problem (1), (2) has some infinite set of solutions.

Proof. - Let $I_{1}, I_{2}$ and $I_{3}$ denote the set of such $i$, for which the conditions 1 ), 2) and 3) are satisfied. Without loss of generality we can suppose that $n \in I_{2} \cup I_{3}$.

Putting

$$
t_{i}^{*}= \begin{cases}t_{i} & \text { for } i \in I_{1} \\ b & \text { for } i \in I_{2} \\ a & \text { for } i \in I_{3}\end{cases}
$$

and

$$
f_{i}^{*}\left(t, y_{1}, \ldots, y_{n}\right)=f_{i}\left(t, y_{1}-x_{01}, \ldots, y_{n}-x_{0 n}\right) \quad(i=1, \ldots, n)
$$

where $x_{0 i}=0(i=1, \ldots, n-1)$ and $x_{0 n}$ is an arbitrary number.

It can be easily verified that on the domain $\mathfrak{D}_{a b}^{n}$ the inequalities

$$
\begin{array}{r}
f_{i}^{*}\left(t, y_{1}, \ldots, y_{n}\right) \operatorname{sign}\left[\left(t-t_{i}^{*}\right)\left(y_{i}-x_{0 i}\right)\right] \leqslant-a_{i}(t)\left|y_{i}-x_{0 i}\right|+\varphi_{i}^{*}(t)\left|y_{i}-x_{0 i}\right|+\psi_{i}(t) \\
(i=1, . ., n) .
\end{array}
$$

hold, where

$$
\varphi_{i}^{*}(t)=\left\{\begin{array}{ll}
\varphi_{i}(t) & \text { for } i \in I_{1} \\
0 & \text { for } i \in I_{2} \cup I_{3},
\end{array} \quad a_{i}(t)=\varphi_{i}(t)-\varphi_{i}^{*}(t)(i=1, \ldots, n) .\right.
$$


Since the diagonal matrix with diagonal elements $\varphi_{1}^{*}(t), \ldots, \varphi_{n}^{*}(t)$ belongs to the set $N_{s}\left(a, b ; t_{1}^{*}, \ldots, t_{n}^{*}\right)$, according to theorem 1.2 the system of differential equations

$$
\frac{d y_{i}}{d t}=f_{i}^{*}\left(t, y_{1}, \ldots, y_{n}\right) \quad(i=1, \ldots, n)
$$

has a solution $y_{1}(t), \ldots, y_{n}(t)$, satisfying the boundary conditions

$$
y_{i}\left(t_{i}^{*}\right)=0 \quad(i=1, \ldots, n)
$$

It is clear that $x_{i}(t)=y_{i}(t)-x_{0 i}(i=1, \ldots, n)$ is a solution of system (1) with the boundary conditions

$$
x_{i}\left(t_{i}^{*}\right)=0 \quad(i=1, \ldots, n-1), x_{n}\left(t_{n}^{*}\right)=-x_{0 n} .
$$

If $i \in I_{2}$, then by 2) we have

$$
\frac{d\left|x_{i}(t)\right|}{d t} \geqslant \varphi_{i}(t)\left|x_{i}(t)\right|-\varphi_{i}(t) \quad \text { for } t_{i}<t<b
$$

and

$$
\left|x_{i}(t)\right| \leqslant \exp \left[-\int_{i}^{b} \varphi_{i}(\tau) d \tau\right]\left\{x_{0 i}+\left[\int_{t}^{b} \varphi_{i}(s) d s\right] \psi_{i}(\tau) d \tau\right\} \rightarrow 0 \quad \text { for } t \rightarrow t_{i}^{*}
$$

In an analogous way we shall prove that $x_{i}\left(t_{i}\right)=0$ for $i \in I_{3}$. Thus for arbitrary $x_{0 n} \in(-\infty,+\infty)$ problem (1), (2) has a solution, satisfying conditions (3.1). Thus the theorem is proved.

COROLLARY. - Let

$$
f\left(t, x_{1}, \ldots, x_{n}\right) \in K\left(a, b ; t_{n}\right)
$$

and let one of the following two conditions be satisfied:

1) $f\left(t, x_{1}, \ldots, x_{n}\right) \operatorname{sign} x_{n} \geqslant \varphi(t)\left|x_{n}\right|-\psi(t)$,

where the functions $p(t) \in L\left(a, b ; t_{n}\right)$ and $\psi(t) \in L(a, b)$ are nonnegative, $a \leqslant t_{n}<b$ and

$$
\int_{t_{n}}^{b} \varphi(\tau) d \tau=+\infty
$$


2) $f\left(t, x_{1}, \ldots, x_{n}\right) \operatorname{sign} x_{n} \leqslant-\varphi(t)\left|x_{n}\right|+\psi(t)$,

where the functions $\varphi(t) \in L\left(a, b ; t_{n}\right)$ and $\psi(t) \in L(a, b)$ are nonnegative,
$a<t_{n} \leqslant b$ and

$$
\int_{a}^{t_{n}} \varphi(\tau) d \tau=+\infty
$$

Then the problem (2.1), (2.2) has an infinite set of salutions.

\section{REFERENCES}

[1] G. A. Bessmerty YCH, Some notes on the problem of existence of a solution for singular systems of ordinary differential equations (Russian), Pribl. Met. Reš. Diff. Uravn., Vip. 2, Kiev, "Naukova Dumka» (1960), pp. 23-30.

[2] J. Gusernov - A. I. Perov, The singular problem of Cauchy for nontinear equations in a Hilbert space (Russian), Uč. zap. Azerb. Un-ta, ser. fiz.-matem.n., no. 3 (1964), pp. 41-50.

[3] I. T. KIGURADZE, On the Cauchy problem for singular systems of ordinary differential equations (Russian), Diff. Uravnenia, 1, no. 10 (1965), pp. 1271-1289.

[4] I. T. Krquradze, The comparison lemma and the question of uniqueness for solutions of the Canchy problem for differential equations (Russian), Soobšč. Akad. Nauk Gruzin. SSR, 39, no. 3 (1965), pp. 513-518.

[5] I. T. Krguradze, On the singular problem of Nicoletti (Russian), Soviet Math. Dokl., 10, no. 3 (1969), pp. 769-772.

[6] A. Lasota, Sur l'existence et l'unicité des solutions au problème aux limites de Nicoletti pour un système d'équations différentielles ordinaires, Zeszyty Nauk, UJ, Prace Mat., 11 (1966), pp. 41-48.

[7] A. Lasota - C. OleCH, An optimal solution of Nicoletti's boundary value problem, Ann. Polon. Math., 18, no. 2 (1966), pp. 131-139.

[8] V. I. Levin, On inequalities, II (Russian), Matem. Sbornik, 9 (46), no. 2 (1938), pp. $309-324$.

[9] 0 . Nrconems, sulle condizioni iniziali ohe determinano gli integrali della equazioni differenziali ordinarie, Atti d. R. Acc. Sc. Torino, 33 (1897-1898), pp. 746-759.

[10] A. I. Prerov, On the singular problem of Canehy (Russian), Trudi Semin. po funk, annal. Voronešskii, Universitet, 7 (1963), pp. 104-107.

[11] A. I. Perov - A. V. KrbenKo, On one general way of studying boundary value problems (Russian), Izv. AN SSSR, Ser. matem., 30, no. 2 (1966), pp. 249-264.

[12] V. P. SKRIPNIK, On one boundary value problem and questions of oscillations of solutions (Russian), Matem. Sbornik, 55 (97), no. 4 (1961), pp. 449-472.

[13] V. A. С̆С̆亡K, The investigation of systems of ordinary differential equations with the singularity (Russian), Trudy Mosk. matem. obšě., 8 (1959), pp. 155-197.

[14] S. CrNQuna, A proposito dell'esistena della soluzione del problema di Nicoleti per sistemi di equazioni differenziali ordinarie, Rend. Ist. Lombardo Accad. Sci. e Lettere, A 101, no. 1 (1967), pp. 3-7.

[15] J. SCHAUDER, Der Fixpunktsatz in Funktionalräumen, Studia Math., 2 (1930), pp. 171-180.

[16] М. Švec, K problému jednoznačnosti integràlov systému lineárnych differenciálnich rovnic, Matem.-Fyz. Sb. SAV, 2, no. 1-2 (1952), pp. 3-22. 